\title{
A New, Efficient Stellar Evolution Code for Calculating Complete Evolutionary Tracks
}

\author{
A. Kovetz ${ }^{1,2}$, O. Yaron ${ }^{1}$ and D. Prialnik ${ }^{1}$ \\ ${ }^{1}$ Department of Geophysics and Planetary Sciences, Sackler Faculty of Exact Sciences \\ ${ }^{2}$ School of Physics \& Astronomy, Sackler Faculty of Exact Sciences, Tel Aviv University, Israel
}

\begin{abstract}
We report on the development of a new stellar evolution code, and provide a taste of results, showing its capability to calculate full evolutionary tracks for a wide range of masses and metalicities. The code is fast and efficient, and is capable of following through all evolutionary phases ,including core/shell flashes and thermal pulses, without any interruption or intervention. It is meant to be used also in the context of modeling the evolution of dense stellar systems, for performing live calculations for both 'normal' ZAMS/PRE-MS models, but mainly for 'noncanonical' stellar configurations (i.e. merger-products). We show a few examples of evolutionary calculations for stellar populations I and II, and for masses in the range $0.25-64 M_{\odot}$.
\end{abstract}

Keywords. stars: evolution, (stars:) Hertzsprung-Russell diagram

\section{The Evolution Code}

[Basic Scheme] The set of differential equations governing the stellar structure and evolution are approximated by finite difference equations and solved by the iterative implicit Newton-Raphson method. Following Eggleton (1971), the equations of structure and composition are all solved simultaneously, together with a mass distribution function - implementing an adaptive mesh. [Input Physics] Equations of state include Coulomb and quantum corrections; $\mathrm{H}$ and He ionization equilibria, $\mathrm{H}_{2}$ creation, pair creation and pressure ionization (Pols et al. (1995)) are taken into account. Using OPAL opacities (Iglesias \& Rogers (1996)) for high temperatures and Ferguson \& Alexander et al. (2005) for low temperatures, and applying electron scattering (for the highest temperatures) and conductive opacities (when applicable), as obtained from Cassisi \& Potekhin et al. (2007), we create, for a required metalicity, our own set of opacity tables, covering both the Hydrogen mass fractions and Carbon/Oxygen excesses mass fractions from 0 to $[1-Z]$. Nuclear reaction rates are from Caughlan \& Fowler (1988), and follow H, $\alpha$ and CO - burning. Neutrino losses are according to Itoh et al. (1996). Several massloss prescriptions have been incorporated; Reimers (1975) and some of it's variations for mostly the advanced (post-RGB) stages. [Computational details] Our automatically varying timesteps, determined mainly by limits imposed on the changes allowed during timestep, span a wide dynamic range - from $\lesssim 1 \mathrm{sec}$ (e.g. core He flash) to $\gtrsim 10^{8}$ years (MS). The grid mass shells span a range of $\sim 10^{-15} M_{\odot}$ (WD atmosphere) to $\gtrsim 10^{-1} M_{\odot}$ (inert stellar core). The typical number of grid points is kept in between 150 and 200; a typical number of timesteps for a complete evolution track is $1-2 \times 10^{3}$; execution time being typically in the range $5-15$ minutes.

\section{Results}

In the four panels of Fig. 1 we show a few representative results of full evolutionary calculations: A calibration solar model, following through all evolutionary stages and 

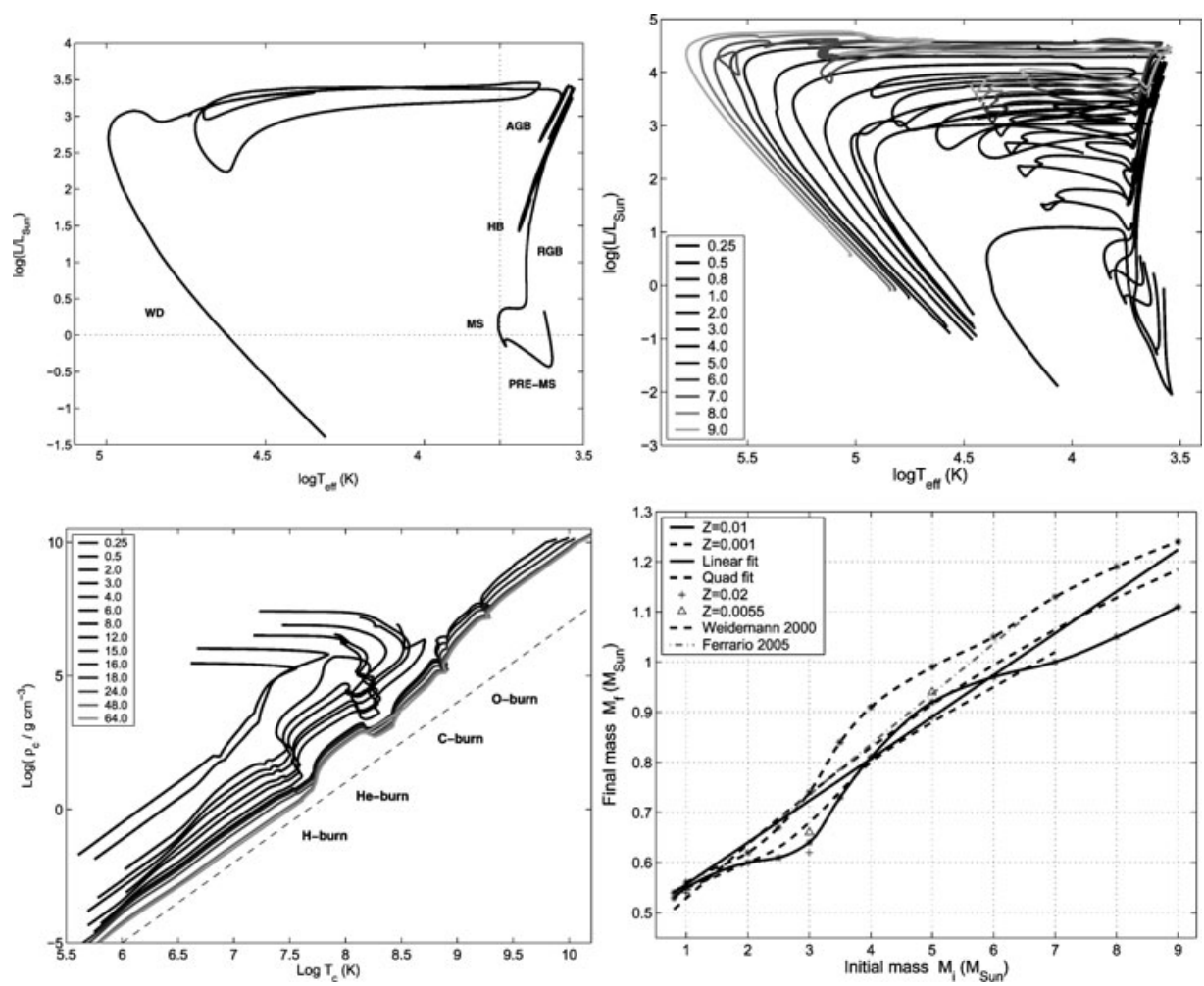

Figure 1. Top left: Solar model $-1 M_{\odot}, Z=0.018, Y=0.29$. Top right: Complete tracks for Pop. II models $(Z=0.001), 0.25-9.0 M_{\odot}$. Bottom left: Pop. I $(Z=0.01)$ - Evolution of the central stellar density and temperature for masses in the range $0.25-64 M_{\odot}$. Dotted line has a slope of 3 (as obtained for the $\log \rho_{c}-\log T_{c}$ relation of hydrostatic equilibrium under ideal gas law). Nuclear burning phases are marked along the tracks. Bottom right: IFMR - Initial vs. final masses for $M_{i}$ in the range $0.8-9 M_{\odot}$, both populations. We show our linear and quadratic fits to all values, and fits of Weidemann 2000 and Ferrario 2005 for comparison.

ending as a cooling $0.55 M_{\odot}$ CO WD. Features at 4.5 Gyr match the present sun to an accuracy of $1 \%$ or better. We show tracks in $\log \rho_{c}-\log T_{c}$ plane, exhibiting the branching off between intermediate-mass stars that end their lives as WDs, and massive stars that go through advanced nuclear burning stages, ending their lives in dynamic core collapse which is followed by the code until very high central pressures are attained and the adiabatic exponent approaches $4 / 3$ throughout the core. Based on our sequences of results for both Pops. I and II, we also present Initial Final Mass Relationship for initial masses in the range $0.8-9 M_{\odot}$, and propose our own fits with comparison to others'.

\section{References}

Cassisi, S., Potekhin, A. Y., Pietrinferni, A., Catelan, M., \& Salaris, M. 2007 ApJ 661, 1094

Caughlan, G. R. \& Fowler, W. A. 1988 Atomic Data and Nuclear Data Tables 40, 283

Eggleton, P. P. 1971, MNRAS 151, 351

Ferguson, J. W., Alexander, D. R., et al. 2005 ApJ 623, 585

Iglesias, C. A. \& Rogers F. J. 1996, ApJ 464, 943

Itoh, N., Hayashi, H., Nishikawa, A., \& Kohyama, Y. 1996 ApJS 102, 411

Pols, O. R., Tout, C. A., Eggleton, P. P., \& Han, Z. 1995, MNRAS 274, 964

Reimers, D. 1975 MSRSL 8, 369 